\title{
Peak to Average Power Ratio Reduction in FBMC Systems by $\mathrm{PN}$-sequences
}

\author{
Duaa Sh. Almomani \\ Department of Electrical Engineering, Jordan University of Science and Technology, Irbid, Jordan
}

\section{Email address:}

dua_mom@hotmail.com

\section{To cite this article:}

Duaa Sh. Almomani. Peak to Average Power Ratio Reduction in FBMC Systems by PN-sequences. Advances in Wireless Communications and Networks. Vol. 3, No. 6, 2017, pp. 84-89. doi: 10.11648/j.awcn.20170306.12

Received: September 25, 2017; Accepted: November 21, 2017; Published: December 14, 2017

\begin{abstract}
In this paper, we propose a method to reduce the peak to average power ratio in filter bank multicarrier systems. The proposed method is based on shuffling the sub-carriers in filter bank multicarrier system randomly, then, Using the PNsequences to shuffle the sub-carriers. The simulation results confirmed the effectiveness of the proposed method and gave observed enhancement.
\end{abstract}

Keywords: Multicarrier Systems, Filter Bank, Peak to Average Power Ratio

\section{Introduction}

While the wheel of telecommunication science development is still flourishing and increasing, the scientists and researchers also are still searching and making their best efforts to put their imprint on science progress to benefit the humanity and make the communication easier between the people.

It is noticeable that in the last two decades, the telecommunication progress has grown hastily, which made the needs of increasing the speed, flexibility for varying traffic conditions, data rate, throughput, and bandwidth in telecommunication transmitting services increase. Multicarrier Modulation (MCM) is considered as one of the methods that used to increase the quality of telecommunication transmission.

There is, however, an important property makes the MCM preferably used in modern telecommunication systems, which is its ability to cope the frequency selectivity. Dividing the wideband frequency selectivity communication channel into several sub-bands makes the probability of having subchannels bandwidth less than the coherence bandwidth is much higher than having entire channel's bandwidth less than the coherence bandwidth. So, the frequency selectivity can be clearly prevented or at least reduced in multicarrier modulation. As a result of that, the throughput will be increased [1].

Orthogonal Frequency Division Multiplexing (OFDM) is a very popular special case of multicarrier system when the sub-carriers are orthogonal to each other. The orthogonality allows high spectral efficiency, because allowing the subcarriers to be overlapped makes OFDM has an efficient use of the spectrum. Thus, almost the whole available frequency band can be utilized. Moreover, it has less sensitivity to sample timing offsets and it converts the channel from selective frequency fading channel to flat fading subchannels by dividing the channel into smaller bands. OFDM system has been using in many current standards because of its efficient implementation with Fast Fourier Transforms (IFFT and FFT) and its simple equalization because of its efficiency to cope the frequency selectivity.

Since the output of the IFFT is the summation of $\mathrm{N}$ modulated sinusoids, it will give high peak power when the number of sub-carriers increases. Adding these sinusoids when they have zero phase difference between each other simultaneously causes a very high peakpower regarding to the mean power for each one alone. Which causes high Peak to Average Power Ratio (PAPR).

Filter Bank Multi-Carrier (FBMC) is another case of $\mathrm{MCM}$, with non-orthogonal carriers (in the frequency domain) and evolved prototype filter which exhibits small ripples in the frequency domain, which makes the wastage of power is less than as it is in OFDM[2]. Moreover, filter bank does not use the cyclic prefix (CP) which allows increasing the utilized bandwidth and so the equalization will be simpler [3]. These attractive features make FBMC more qualified to 
applied in the coming telecommunication generations.

Unfortunately, both OFDM and FBMC systems suffer from PAPR. Actually, PAPR is considered one of the main problems which face multicarrier systems.

The main objective of this paper is to suggest a method that may reduce the PAPR in FBMC system. This method is based on shuffling the subcarriers to reduce the possibility of constructive subcarriers summation, therefore reduce the PAPR.

The second section of this paper talks briefly about the previous researches and methods that were proposed to reduce the PAPR. Section three clarifies the PAPR problem in FBMC. Section four proposes the modeling of our suggested method, section five shows the simulation results for this method, and finally the last section concludes the observations of this work and the future works that may be applied depending on this work.

\section{Literature Review}

Many of previous techniques have been done to reduce PAPR in MCM systems, both OFDM and FBMC systems. Some of the main techniques that were used in OFDM are; Clipping [4], Coding [5], interleaving [6], Partial Transmit Sequence (PTS) [7], Selecting Mapping (SLM) [8], Tone Injection (TI) [9], Tone Reservation (TR) and Active Constellation Extension (ACS) [10].

Some of the solutions that were used to reduce PAPR in OFDM systems cannot be applied for FBMC, like Coding, Interleaving, PTS, SML and TI. And this is due to the overlapping between FBMC symbols in the time domain [11].

Since FBMC is a new candidate to be used in the next generations, the researches are stillimited beyond this technology. Therefore, alittle bit of methods have been done to reduce the PAPR. Clipping is the simplest method hat used for this purpose in FBMC systems [12]. The discrete amplitude of the time domain FBMC signal is limited to a threshold, such that the signal above this threshold will be cut away. The disadvantage of this method is it introduces in-band and outband distortion, which will affect the system performance.

Active Constellation Extension (ACE) is an efficient algorithm proposed by Krongold and Jones [13], it reduces PAPR in FBMC systems by correcting the distorted constellations after clipping the time domain signal, such that keeping the minimum Euclidean distance. In companding method, the small signals being enlargedand the large signals being compressed to save the small signals from noise. Two types of companders A-law Companding and $\mu$-law companding; where $\mathrm{A}$ and $\mu$ are compression parameters, the previous works for these two types showed that both companders have almost the same performance in reducing PAPR. Another work has been done by using clipping technique and companding together [14], and it has been shown that A-low companding with clipping method outperforms the performance $\mu$-law companding with clipping in PAPR reduction.

As mentioned before, the Tone Reservation (TR) is not an efficient method to reduce PAPR in FBMC systems due to the overlapping fact in the time domain in FBMC systems. Thus, Sliding Window is another approach added to the TRtechnique made TR with sliding window can be applied in FBMC systems with good results [15].

\section{Peak to Average Power Ratio in FBMC Systems}

Due to the overlapping nature between the subcarriers in FBMC, the PAPR for an FBMC symbol is more complicated to analyze. The length of the FBMC impulse response (L) must be $\mathrm{K}$ times of the number of the subcarriers (i.e. $\mathrm{L}=\mathrm{KN}$ ), where $\mathrm{K}$ is the number of overlapped subcarriers.

Referring to [16], the OQAM/FBMC baseband may be written as:

$$
\mathrm{S}(\mathrm{t})=\sqrt{2} \sum_{m=-\infty}^{\infty} \sum_{n=0}^{2 N-1}\left(\mathrm{a}_{\mathrm{m}, \mathrm{n}} \mathrm{p}(\mathrm{t}-\mathrm{mT})\right) \cdot e^{j 2 \pi(2 n) \Delta f t} e^{j \varphi_{m \cdot n}}
$$

Where $\mathrm{N}$ is the number of FBMC subcarriers, $\mathrm{m}$ is the running time and the separation between two successful subcarriers $\Delta \mathrm{f}=1 / \mathrm{NT}=1 / \mathrm{T}_{\mathrm{s}} . \mathrm{T}_{\mathrm{s}}$ is the OFDM symbol duration.

After applying filtering process, the symbols will be extended to a length of $\mathrm{L}=\mathrm{KN}$. The OQAM FBMC symbols are staggered by $\mathrm{N} / 2$ samples apart to compensate for OQAM modulation and the extended symbol length.

To find the PAPR in FBMC, the FBMC overlapped symbols cannot be denied. Therefore, let $\mathrm{M}$ be a frame containing the overlapped FBMC symbols over which the PAPR can be accurately measured. Thus, two different measurements of PAPR can be obtained for the FBMC, the first measurement contains the main all symbols and the second one contains the overlapped symbols frame with length $(\mathrm{M}-1) \mathrm{N} / 2+\mathrm{L}$, the frame-based PAPR can be defined with $\mathrm{s}[\mathrm{n}]$ the discrete samples as [11]:

$$
\operatorname{PAPR}\left(s_{m}\right)_{\mathrm{dB}}=10 \log \left(\frac{\left.\max _{0 \leq t \leq(M-1) N / 2+L-1}\right|^{2}}{E\left[\left|s_{m}\right|\right]^{2}}\right)
$$

Where $s_{m}=[\mathrm{s}[0], \ldots, \mathrm{s}[(\mathrm{M}-1) \mathrm{N} / 2+\mathrm{L}-1]]^{\mathrm{T}}$. For the main symbols, the PAPR will be:

$$
\operatorname{PAPR}\left(s_{v}\right) \mathrm{dB}=10 \log \left(\frac{\max _{0 \leq t \leq N-1} \mid s_{v}^{2}}{E\left[\left|s_{v}\right|\right]^{2}}\right)
$$

Wheres $_{v}=[s[L-N / 2+v N], \ldots, s[L-N / 2+(v$ + 1) $N-1]] T$.

Here new symbols $s_{v}$ are defined, over which the PAPR can be measured. And these symbols are broken up from the modulated signal $s[n]$ to measure the accurate PAPR that be equivalent with the OFDM systems. Where the PAPR cannot be measured at the start and end of an FBMC frameaccurately. So, the PAPR should be measured within a full filter at the start of FBMC frame and concluding a full filter length before the end of the frame.

To examine the PAPR reduction, finding the probability when the PAPR exceeds specific value is needed. In other 
words, finding the Complementary Cumulative Distribution function (CCDF) at different values of PAPR. Whereas $\mathrm{CCDF}$ function indicates how much the time signal remains at or exceeds a given power level.

considering $\mathrm{N}$ is large, the central limit theorem can be invoked, therefore, the real and Imaginary part of the signal can be considered asymptotically Gaussian. Under this assumption, the envelop of the signal follow Rayleigh distribution and the power follows Exponential probability density function. The Cumulative Distribution Function (CDF) for the Exponential distribution is given by:

$$
\mathrm{F}(\mathrm{z})=\left(1-e^{-z}\right)
$$

CDF shows the probability that the signal with $\mathrm{N}$ subcarriers is less than specific value $(z)$, which is the probability that all subcarriers are less than power level (z) assuming that the subcarriers are statically independent;

$$
\mathrm{F}(\mathrm{z})=\mathrm{P}(\mathrm{Z}<\mathrm{z})=\mathrm{P}\left(Z_{1}<\mathrm{z}\right) \cdot \mathrm{P}\left(Z_{2}<\mathrm{z}\right) \ldots \mathrm{P}\left(Z_{N-1}<\mathrm{z}\right)
$$

The CDF for N-subcarriers follow Exponential distribution is given by:

$$
\mathrm{F}(\mathrm{z})=\left(1-e^{-z}\right)^{N}
$$

The Complementary Cumulative Distribution Function $(\mathrm{CCDF})$ is:

$$
\begin{aligned}
\bar{F}(\mathrm{z}) & =\mathrm{P}(\mathrm{Z} \geq \mathrm{z})=1-\mathrm{P}(\mathrm{Z}<\mathrm{z}) \\
& =1-\left(1-e^{-z}\right)^{N}=\mathrm{P}(\mathrm{PAPR} \geq \mathrm{z})
\end{aligned}
$$

subcarriers $\mathrm{N}$ increases the $\mathrm{P}(\mathrm{PAPR} \geq \mathrm{z})$.

\section{System Modeling}

The proposed FBMC transmitter contains a synthesis filter bank (SFB) and the receiver contains an analysis filter bank (AFB). In the structure of figure 1, the Inverse Fast Fourier Transform (IFFT) is presented as the same as in OFDM with extra Poly-Phase Network (PPN) filters which consists of a set of digital filters with specific coefficients, resulting from the impulse response of the prototype low pass filter.

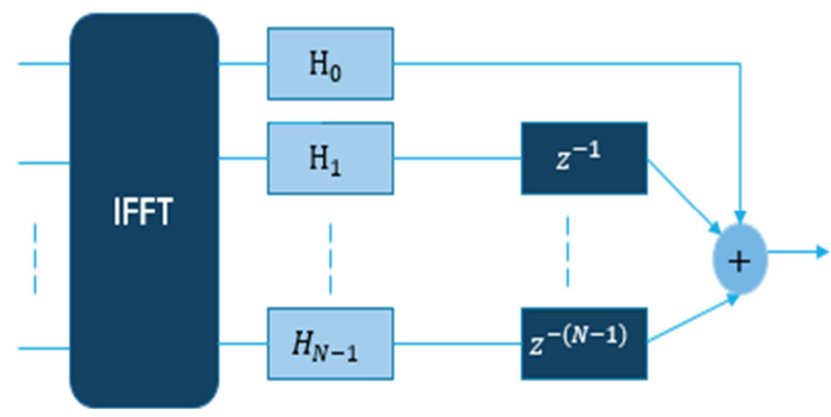

Figure 1. The transmitter block diagram.

The major idea in this work is to prove that shuffling the subcarriers in FBMC systems may reduce the PAPR. Two suggested proposed systems were applied; the first system depends on shuffling the subcarriers in FBMC system randomly, and the second one is about shuffling the subcarriers based on $\mathrm{PN}$-sequences.

From equation (7) it is clear that increasing the number of

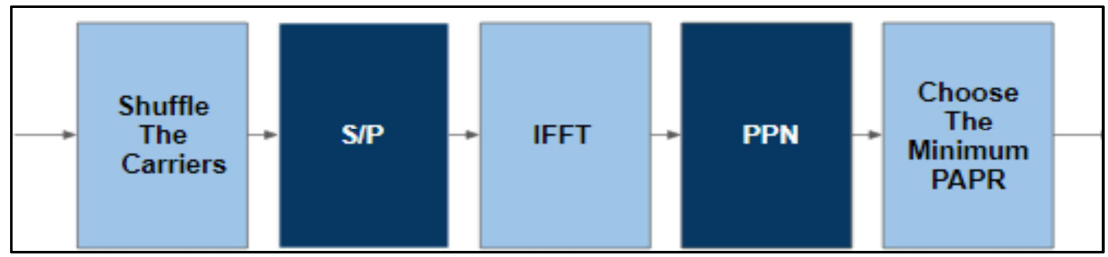

Figure 2. The proposed algorithm block diagram.

The goal of this work is to propose a method that reduces the PAPR in FBMC systems by trying to reduce as much as possible the constructively summation of the subcarriers signals. The default set of the subcarriers is $\{0, \Delta f, 2 \Delta f$, $3 \Delta f, \ldots,(\mathrm{N}-1) \Delta f\}$, and in this section, the default order of the subcarriers was shuffled randomly, then the PAPR for the new order was measured.

The proposed method was tested using Matlab simulation with full FBMC system implementation. PHYDYAS Bellanger prototype filter [17] was chosen, several number of subcarriers were tested with different values of OQAM modulation schemes.

The QAM symbols were generated by Rectangular QAM Modulator object tool in matlab, which modulates M-ary quadrature signal. amplitude modulation with a rectangular constellation. The output is the baseband of the modulated signal. This tool is applied after calling it by "step" keyword in the code.

Then, the QAM symbols staggered by $\mathrm{T} / 2$, in other words, the odd and even QAM symbols alternate into real and imaginary parts. The symbols after that were shuffled, converted from serial to parallel sequences to apply the IFFT process; where the sequences here are treated as frequency domain not time the output then would be multiplied by the polyphase network (PNN), which is PHYDYAS Bellanger filters [17] in our case.

\section{Simulation Results}

Our work for PAPR measurements is based on the consideration of the overlapped region in FBMC symbols, then after specific iterations of shuffling, the least PAPR is chosen. Finally, the CCDF respect to the PAPR is drawn to fulfill the performance of the proposed method. 


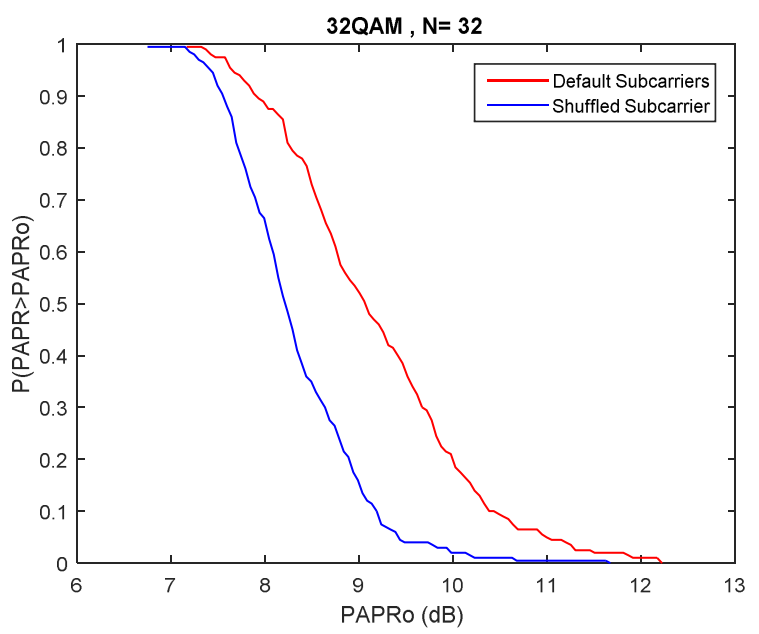

Figure 3. The CCDF for two FBMC systems with default set of subcarrier frequencies and a shuffled set of subcarrier frequencies when $N=32$.

Figure 3 illustrates the enhancement in the system performance after shuffling the subcarriers when the number of subcarriers (N) is 32 and the modulation is 32 offset QAM. Where the shuffled set of subcarriersgives lower PAPR than the default set of subcarriers.

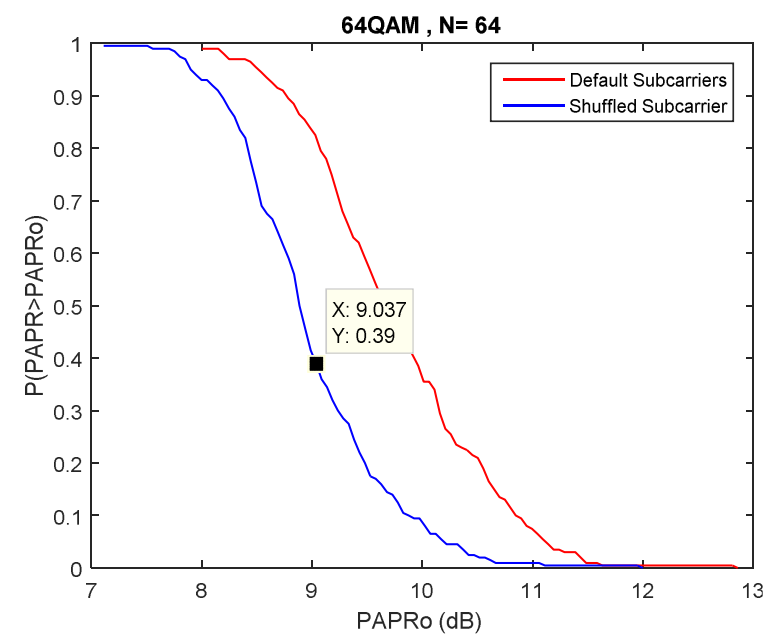

Figure 4. The CCDF for two FBMC systems with default set of subcarrier frequencies and a shuffled set of ubcarrier frequencies when $N=64$.

Figure 4 for example shows the system performance when the number of subcarriers is 64 . For the default set of the subcarriers, the probability of having PAPR more than $9 \mathrm{dBis}$ 0.825 while it is 0.39 when the PAPR is above almost $9 \mathrm{~dB}$ for the shuffled subcarriers. Hence, the improvement is achieved.

The shuffled carriers proved their efficiency to reduce the PAPR in FBMC systems, and the results in the previous section gave a clear improvement. But unfortunately shuffling the carriers randomly without fixed standards will not be efficient at the receiver side. As it is known that the receiver needs an ordered standard to reconstruct the transmitted signal with the least possible errors, which makes random shuffling is not a practical solution. Hence, using a Pseudo-Noise (PN) sequence was suggested to solve this problem, whereas the PN sequences go through a uniform order, and this order can be provided at the receiver side.

The PN sequence consists of 1's and 0's, the sequence will repeat itself after its period, so it looks randomly for the user who does not know the code, while it will be deterministic and known for both the transmitter and the receiver since they already know the code.

The simulation gave good results with obvious improvement at specific connections and specific initial values as well. For example, for 32 subcarriers which means 4 digits PN shift register some of taps connections gave better performance and other connections gave worse performance. Which means that the choice of the connections must be selected carefully, moreover, the initial input changes the order and hence the performance will be affected.

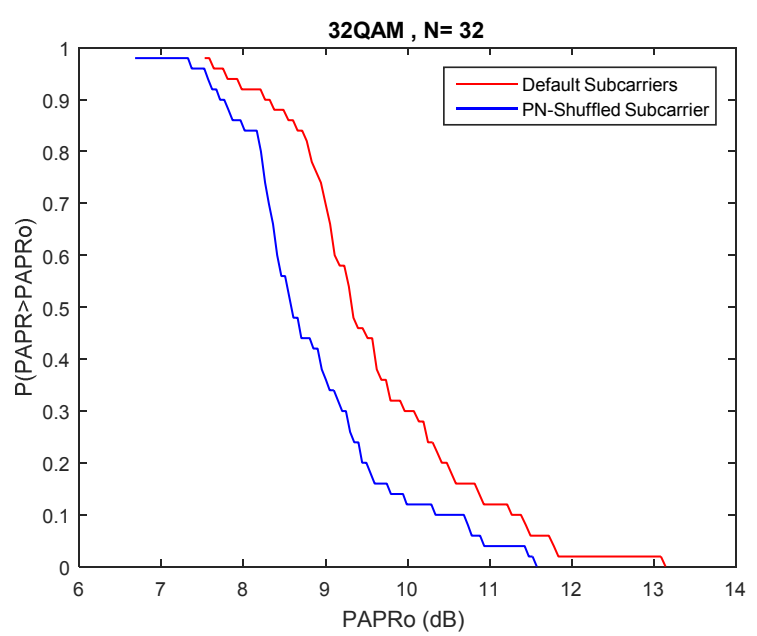

Figure 5. The CCDF for two FBMC systems with default set of subcarrier frequencies and a PN-shuffled set of subcarrier frequencies when the connection is (0 100 1), initial input 16and $N=32$.

Figure 5 illustrates the performance when the connection is $\left(\begin{array}{lllll}0 & 1 & 0 & 0 & 1\end{array}\right)$ and the initial shift register input number is 16 . We can note from this figure that the performance of the PNshuffled set of subcarriers set gives lower PAPR than the default set of subcarriers.

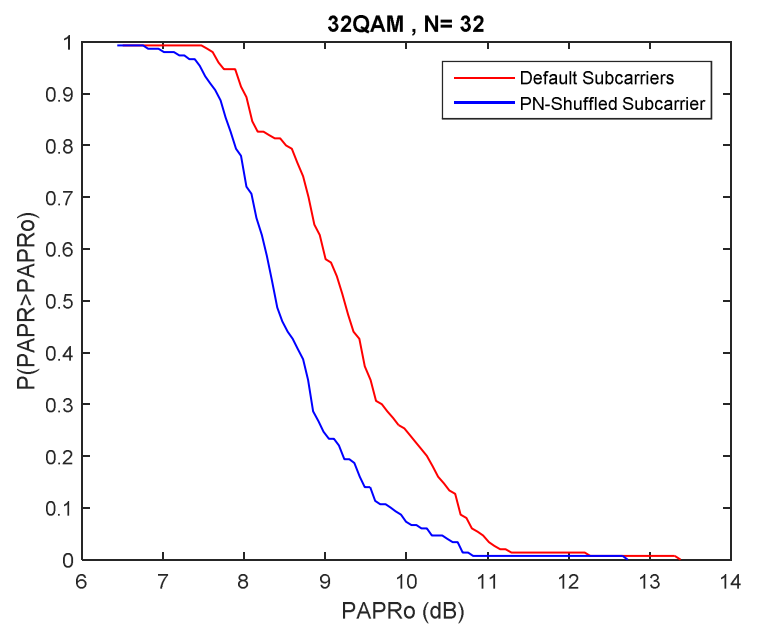

Figure 6. The CCDF for two FBMC systems with default set of subcarrier frequencies and a $P N$-shuffled set of subcarrier frequencies when the connection is (0 1001 ), initial input 19 and $N=32$. 
Moving on to 6-digits PN shift register, which can shuffle 64-subcarriers since the number of the subcarriers is given as the equation (8). while the maximum length of the register output is 63 , and this means that the last subcarrier stays in the same original order.

$$
\mathrm{N}=2^{\mathrm{m}}
$$

This connection $\left[\begin{array}{llllll}0 & 1 & 1 & 0 & 1 & 1\end{array}\right]$ is one of the connections that improved the system performance, and the following figure show the enhancement.

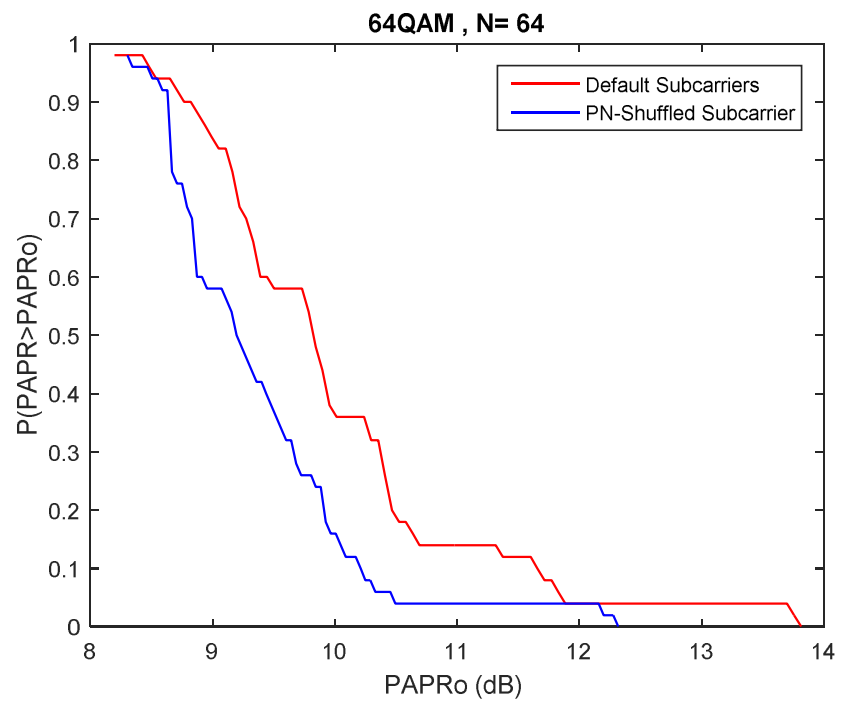

Figure 7. The CCDF for two FBMC systems with default set of subcarrier frequencies and a PN-shuffled set of subcarrier frequencies when the connection is (0 1110011$)$, initial input 11 and $N=64$.

when $\mathrm{N}=64$, which means 6 -digits shift register is used, the system is still giving better performance at specific connections and at specific initial value. Figure 8 shows the enhancement when these taps [ $\left[\begin{array}{lllllll}0 & 1 & 1 & 1 & 1 & 1 & 1\end{array}\right]$ are connected with initial value equal 125 .

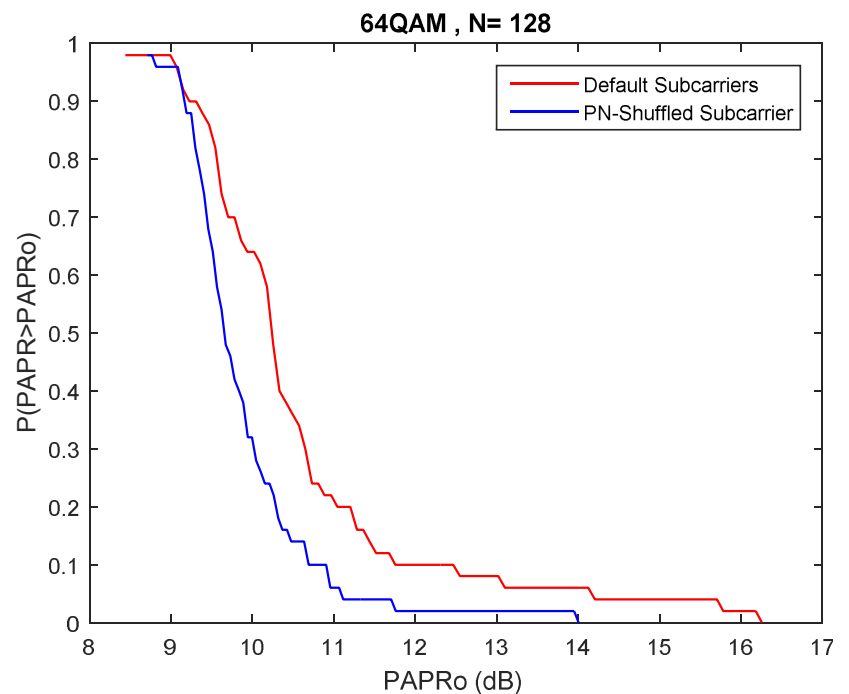

Figure 8. The CCDF for two FBMC systems with default set of subcarrier frequencies and a PN-shuffled set of subcarrier frequencies when the connection is (0 1111111 ), initial input 125 and $N=64$.
The system keeps giving good enhancement with several values of OQAM modulation, subcarriers number and with different PN- sequences.

\section{Conclusion}

In this paper, a new technique has been proposed to reduce PAPR. The main concept of this technique depends on shuffling the default order set of subcarriers to obtain a new shuffled set that can reduce PAPR. The choice of shuffling is to decrease the possibility of peaks of the subcarriers signals to occur simultaneously. An idea to shuffle the subcarriers by prearranged order was proposed. It depends on shuffling the set of the subcarrier using PN-sequences, in this process the subcarriers will have the PN shift register output. Both of shuffling methods; randomly and using PN-sequences confirmed good performance and the simulation results show the improvement.

This work may open the doors for future researches focus on shuffling property to reduce PAPR in FBMC systems using another sequences and codes with better performance and improvement.

\section{References}

[1] Hidalgo Stitz, T. (2006). Channel Equalization in Filter Bank Based Multicarrier Modulation for Wireless Communications. 1st ed. BioMed Central Ltd.

[2] Raju, N. and Pillai, D. Companding and Pulse Shaping Technique For PAPR Reduction in FBMC Systems. In: 2015 International Conference on Control, Instrumentation, Communication and Computational Technologies (ICCICCT), 2015.

[3] Hoeg, W. and Lauterbach, T. 2009. Digital audio broadcasting. 1 st ed. Hoboken, New Jersey: John Wiley \& Sons.

[4] Kollár, Z. and Horváth, P. (2012). PAPR Reduction of FBMC by Clipping and Its Iterative Compensation. Journal of Computer Networks and Communications, 2012, pp.1-11.

[5] Jiang, W. and Wang, K. (2013). An Improved PAPR Suppression Algorithm based on Channel Coding in OFDM System. Journal of Networks, 8(11).

[6] Sakran, H., Shokair, M. and Elazm, A. (2009). COMBINED INTERLEAVING AND COMPANDING FOR PAPR REDUCTION IN OFDM SYSTEMS. Progress In Electromagnetics Research C, 6, pp.67-78.

[7] PAPR reduction in OFDM system with Wavelet transformation and partial transmit sequence technique. (2017). Journal of Advances in Technology and Engineering Research, 3(1).

[8] Sohn, I. (2007). RBF Neural Network Based SLM Peak-toAverage Power Ratio Reduction in OFDM Systems. ETRI Journal, 29(3), pp.402-404.

[9] Lee, W., Choi, J. and Huynh, C. A modified tone injection scheme for PAPR reduction using genetic algorithm. ICT Express 2015, 1(2), pp.76-81. 
[10] Chen, C. and Wu, J. PAPR reduction scheme with selective tone reservation for OFDM signals. International Journal of Communication Systems 2012, 26(9), pp.1196-1205.

[11] Van der Neut, N., Maharaj, B., de Lange, F., González, G., Gregorio, F. and Cousseau, J. (2014). PAPR reduction in FBMC using an ACE-based linear programming optimization. EURASIP Journal on Advances in Signal Processing, (1).

[12] Kollár, Z., Varga, L., Horváth, B., Bakki, P. and Bitó, J. (2014). Evaluation of Clipping Based Iterative PAPR Reduction Techniques for FBMC Systems. The Scientific World Journal, 2014, pp.1-12.

[13] Krongold, B. and Jones, D. (2003). Par reduction in ofdm via active constellation extension. IEEE Transactions on Broadcasting, 49(3), pp.258-268.
[14] Wang, Z. (2011). Combined DCT and Companding for PAPR Reduction in OFDM Signals. Journal of Signal and Information Processing, 02(02), pp.100-104.

[15] Lu S, Qu D, He Y: Sliding window tone reservation technique for the peak-to-average power ratio reduction of FBMCOQAM signals. IEEE Wireless Commun-Lett 2012, 1(4):268271. doi:10.1109/WCL.2012.062512.120360.

[16] Siohan, P., Siclet, C. and Lacaille, N. (2002). Analysis and design of OFDM/OQAM systems based on filterbank theory. IEEE Transactions on Signal Processing, 50(5), pp.1170-1183.

[17] Bellanger, M. and Daguet, J. (1974). TDM-FDM Transmultiplexer: Digital Polyphase and FFT. IEEE Transactions on Communications, 22(9), pp.1199-1205. 